\title{
Melt viscosities of lattice polymers using a Kramers potential treatment.
}

\author{
O. Dürr ${ }^{1}$, H.L. Frisch ${ }^{2}$ and W. Dieterich ${ }^{1}$ \\ ${ }^{1}$ Fachbereich Physik, Universität Konstanz, D-78457 Konstanz, Germany \\ ${ }^{2}$ Dept. of Chemistry, SUNY at Albany, Albany NY 12222, USA
}

\begin{abstract}
Kramers relaxation times $\tau_{K}$ and relaxation times $\tau_{R}$ and $\tau_{G}$ for the end-to-end distances and for center of mass diffusion are calculated for dense systems of athermal lattice chains. $\tau_{K}$ is defined from the response of the radius of gyration to a Kramers potential which approximately describes the effect of a stationary shear flow. It is shown that within an intermediate range of chain lengths $N$ the relaxation times $\tau_{R}$ and $\tau_{K}$ exhibit the same scaling with $N$, suggesting that $N$-dependent melt-viscosities for non-entangled chains can be obtained from the Kramers equilibrium concept.
\end{abstract}

\section{INTRODUCTION}

Extending the novel approach by Kramers $\$$ for polymeric solutions under shear (based on replacing the simple shear flow by its irrotational part) 2 t to melts allows us to compute a characteristic time $\tau_{K}$ related to the melt viscosity. In Ref. 5 the method was applied to alkane chains up to about 50 carbon atoms. In this note we compare this concept for melts with calculations of characteristic times for the end-to-end relaxation of chains (rotational time $\tau_{R}$ ) and a time $\tau_{G}$ obtained from the center of mass diffusion. This is achieved by Monte Carlo simulations for dense systems of self-avoiding lattice chains on a simple-cubic lattice. Chains up to a length $N=120$ are considered, which near the upper limit already indicate the onset of entanglements. Our calculations show that there exists an intermediate range of chain lengths $N$ where $\tau_{K}$ and $\tau_{R}$ agree in their dependence on $N$. This confirms the conclusion that important information on the melt viscosity as a function of $N$ can be drawn from knowledge of fourth and sixth moments of the equilibrium distribution function for beads of the chain. In the next section we describe our lattice model, followed in section III by the description of our relaxation time results.

\section{LATTICE-MODEL AND KRAMERS RELAXATION TIME $\tau_{K}$}

We model the self-avoiding polymer by athermal lattice chains where every point of a simple cubic lattice with unit spacing is allowed to be occupied by at most one chain monomer. In this way the hard-core repulsion is taken into account. For the dynamics and for the equilibration of our system we use the generalized Verdier-algorithm, 6 consisting of local moves of one or two monomers. This allows us to determine the center of mass coordinates, the center of mass diffusion constant and the end to end distance as a function of Monte-Carlo time steps, for a number of chain densities. The average chain densities are measured by the number of monomers per lattice point. We investigate monomer densities $n$ ranging from a single chain up to $n=0.5$ (half filled lattice).
In Ref. 2 it was shown that a relaxation time $\tau_{K}$ can be obtained from the Kramers potentiall for Rouse chains in a simple shear flow. This time is obtained from the equilibrium averaged fourth and sixth moments of chain monomer coordinates $x_{i}$ and $y_{i}$ relative to the center of mass. If $\left\langle X_{G}^{2}\right\rangle_{o}$ is the mean squared $\mathrm{x}$-component of the radius of gyration of the chain at equilibrium, thent

$$
\begin{array}{r}
\tau_{K}^{2}=\frac{1}{2}\left[\left\langle X_{G}^{2}\right\rangle_{o}^{-1} \frac{1}{N} \sum_{i=1}^{N} \sum_{j=1}^{N} \sum_{k=1}^{N}\left\langle x_{i}^{2} x_{j} x_{k} y_{j} y_{k}\right\rangle_{0}-\right. \\
\left.\sum_{j=1}^{N} \sum_{k=1}^{N}\left\langle x_{j} x_{k} y_{j} y_{k}\right\rangle_{0}\right]
\end{array}
$$

where $x_{i}$ and $y_{i}$ are the coordinates of the chain monomers with respect to the center of mass of a chain. In the Rouse model one can calculate the shear viscosity $\eta$ from this relaxation time using

$$
\eta=\frac{\pi^{2} \rho N_{A} k_{B} T \tau}{12 M}
$$

where $\rho$ is the mass density, $N_{A}$ is Avogadro's number, $M$ the molar mass of the molecule and $\tau$ either the Rouse or the reduced Kramers time given by (11). With $\tau$ equal to the reduced Kramers time $\tau_{K}$, Eq. 2 was shown earlier: to give the melt (shear) viscosity of shorter alkanes up to about $50 \mathrm{CH}_{2}-$ units. In this note we do not obtain $\eta$ directly but we calculate and compare $\tau_{K}, \tau_{R}$ and $\tau_{G}$ as a function of chain length $N$ at various monomer densities. While $\tau_{K}$ is calculated from (1), we obtain $\tau_{R}$ by fitting an exponential to the decay of the end-to-end vector correlation function at large times. The time $\tau_{G}=\left\langle R_{G}^{2}\right\rangle / D$ is obtained from simulated center of mass diffusion coefficients D and the mean square radius of gyration $\left\langle R_{G}^{2}\right\rangle$.

\section{SIMULATION RESULTS FOR $\tau_{K}, \tau_{R}$ AND $\tau_{G}$}

As a background to our further results we show in Fig. 1a plot of the diffusion constants $D$ divided by the Rouse diffusion coefficient $D_{0}=(1-n) / N$ for various densities. Here the factor $1-n$ has been introduced to 
account for the average effect of blocking of sites by other monomers. The drop in $D / D_{o}$ for sufficiently large $N$, seen in Fig. 1, can be thought to herald the onset of reptation. The horizontal line represents the expected $N$ dependences of the free chain while the line with slope -1 reflects the expected behavior for reptating chains. The inset provides an approximate master curve for the diffusion constant versus $N / N_{e}$ where $N_{e}$ is an estimated length for the onset of entanglements. 8

Fig. 2 presents double-logarithmic plots of the various relaxation times against $N$ for three different densities. Naturally, for short chains one wouldn't expect any universal properties so that differences in the behaviors of $\tau_{K}, \tau_{R}$ and $\tau_{G}$ are not surprising. In the dilute limit, displayed in Fig. 2a, both $\tau_{K}$ and $\tau_{R}$ grow with $N$ approximately as $(N-1)^{2.26}$ when $N \gtrsim 20$, in reasonable agreement with earlier numerical studies. 20. 10 For the purpose of illustrating this $N$-dependence common to $\tau_{K}$ and $\tau_{R}$ we have multiplied $\tau_{K}$ by a constant factor so that both sets of data fall on a common curve. The associated effective exponent in this range of $N$-values is in fair agreement with the theoretical expectation $\tau_{R} \sim N^{2 \nu+1}$ (see Ref. 11) with $\nu \approx 0.59$. For comparison, data for $\left\langle R_{G}^{2}\right\rangle / N \sim N^{2 \nu-1}$ are also shown in Fig. 2a. On the other hand, with $D \sim N^{-1}$ for unentangled chains, it is clear that $\tau_{G}$ will also be proportional to $N^{2 \nu+1}$.

A different behavior is observed in Fig. $2 \mathrm{~b}$ for $\mathrm{a}$ monomer density $n=0.3$. Apart from a constant numerical factor, the relaxation times $\tau_{K}$ and $\tau_{R}$ fall together in the range $10 \lesssim N \lesssim 10^{2}$. Deviations beyond $N \simeq 10^{2}$ are expected to be caused by entanglement effects which then are no longer negligible and are not included in the Kramers method. Note that for large $N$ sufficiently above the entanglement length one expects $\tau_{R} \sim N^{3.4}$ and $\tau_{G} \sim N^{3}$. The effective dynamic exponent read from the slope of the straight line in Fig. 2b in principle may depend on $n$ (see Ref. 10) but, similar to Fig. $2 \mathrm{a}$, is still found in fair agreement with $2 \nu+1$. On the other hand, screening effects now diminish the growth of $\left\langle R_{G}^{2}\right\rangle$ with $N$ relative to the case of a free chain shown in Fig. 2a. The stronger increase of $\tau_{G}$ with $N$ becomes clear from the behavior of $D$, see Fig. 1.

These trends with $n$ are continued in Fig. 2c where the range of agreement between $\tau_{K}$ and $\tau_{R}$ shrinks to about $10 \lesssim N \lesssim 30$. Fig. $2 \mathrm{c}$ also suggests that for $N$ substantially larger than $N_{e} \tau_{K}$, assumes again Rouse-like scaling behavior, i.e. $\tau_{K} \sim N^{2}$.

In passing, we note that for the ratio of the square of the end-to-end distance $R$ to the squared radius of gyration $R_{G}$ saturates with $N$ and comes close to the value 6 for our higher densities, as expected for Gaussian chains, whereas for lower densities this ratio is significantly larger than 6 . This ratio versus $N$ is plotted in Fig. 3 .

\section{CONCLUSION}

The Kramers potential approach for the viscosity relaxation time of dense systems of lattice chains has been verified numerically for an intermediate range of chain lengths. For longer chains the breakdown of this concept could be identified as a result of the onset of entanglement effects. Generally, the Kramers approach offers the possibility to obtain information on a dynamic quantity from equilibrium data. Equilibrium lattice chain configurations can be generatedvery efficiently via the configurational bias method.12 From such data the static moments in Eq. 1 and hence information about melt viscosities in a certain range of densities and chain lengths can be obtained with minimal computational effort.

\section{ACKNOWLEDGMENTS}

We acknowledge helpful discussion with F. Eurich and P. Maass. H. L. Frisch was supported in part also by the NSF grant DMR 9623224, the Humboldt Foundation and Donors of the Petroleum Fund of the American Chemical Society.

${ }^{1}$ H. A. Kramers, J. Chem. Phys. 14, 615 (1946)

${ }^{2}$ H. L. Frisch, N. Pistor, A. Sariban and K. Binder, J. Chem. Phys. 89, 5194 (1988)

${ }^{3}$ K. Ohno, M. Schulz, K. Binder And H. L. Frisch, J. Chem. Phys. 101 (5), 4452 (1994)

${ }^{4}$ H. L. Frisch, M. Schulz and P. R. Srikanth Sharma, Computational Polymer Sci 4, 13 (1994)

${ }^{5}$ W. Paul and H. L. Frisch, Phys. Rev. E 60, 697 (1999)

${ }^{6}$ P.H. Verdier J. Comput. Phys., 4, 227 (1969)

${ }^{7} \mathrm{Up}$ to a numerical factor, the time unit is $\zeta / k_{B} T$, where $\zeta$ is the friction coefficient in the equivalent Rouse model.2

${ }^{8}$ W. Paul, K. Binder, D. W. Herrmann and K. KreMER, J. Phys. II (France) 1, 37 (1991)

${ }^{9}$ K. Binder in Monte Carlo and Molecular Dynamics Simulations in Polymer Science, edited by K. Binder (Oxford University Press, 1995).

${ }^{10}$ J. Skolnick And A. Kolinski, Avd. Chem. Phys. 78, 223 (1990)

${ }^{11}$ P. G DE Genns Scaling Concepts in Polymer Physics (Cornell University Press, 1979)

12 J. Siepmann And D. Frenkel, Mol. Phys. 75, 59 (1992) 
FIGURES

Fig. 1. Chain-length dependence of the diffusion coefficient for a free chain $(\square), n=0.1(*), n=0.3(\times)$ and $\mathrm{n}=0.5(+)$. By using $D_{0}^{*}=k^{*} / N$ with $k^{*}$ being chosen as a free parameter for each $n$ and by rescaling the $\mathrm{N}$ axis via $N \rightarrow N / N_{e}$ all curves (except the one for free chains) fall together on one curve, as shown in the inset. The values chosen for $N_{e}$ are 23 for $n=0.5,50$ for $n=0.3$ and 150 for $n=0.1$ (and $k^{*}=0.50,0.72,1.28$ respectively).

Fig. 2. Comparison of the reduced Kramers relaxation times $\tau_{K} /(N-1)^{2} \times$ with the relaxation time of the end-to-end vector $\tau_{R} /(N-1)^{2}(\square)$ and $\tau_{G} /(N-1)^{2}$ (*) for different number densities $n$, a) free chains, b) $n=0.3$ and c) $n=0.5$. Also included is the radius of gyration squared $(+)$ divided by $N-1$. Typical error bars for larger $N$-values are indicated in Fig. 2c, whereas the size of error bars in the intermediate range, where $\tau_{R}$ and $\tau_{K}$ coincide, is comparable to or smaller than the symbol size.

Fig. 3. Comparison of $R^{2} / R_{G}^{2}$ vs. chain length $N$ for different concentrations. Also included is the $N \rightarrow \infty$ limit for Gaussian chains.

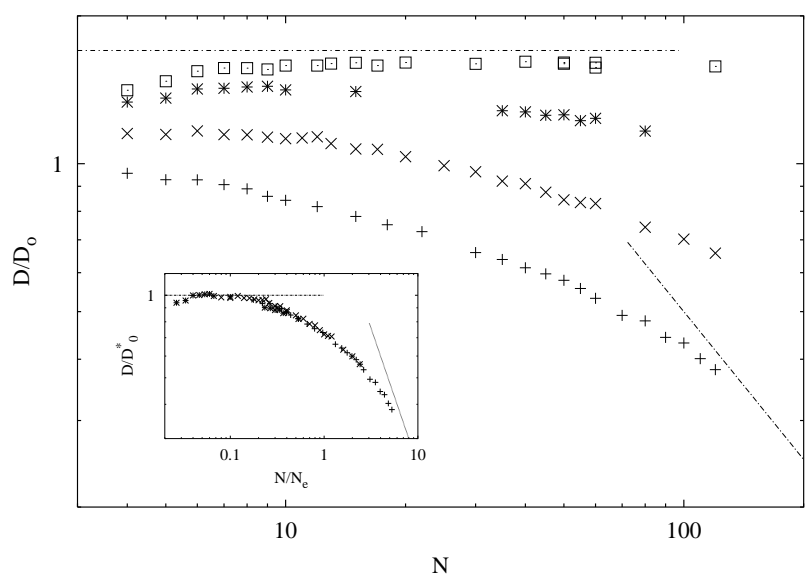

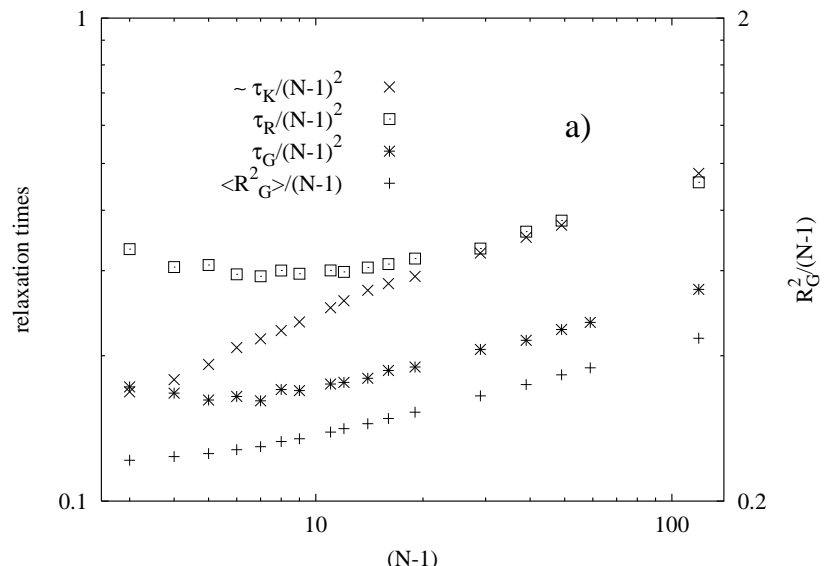
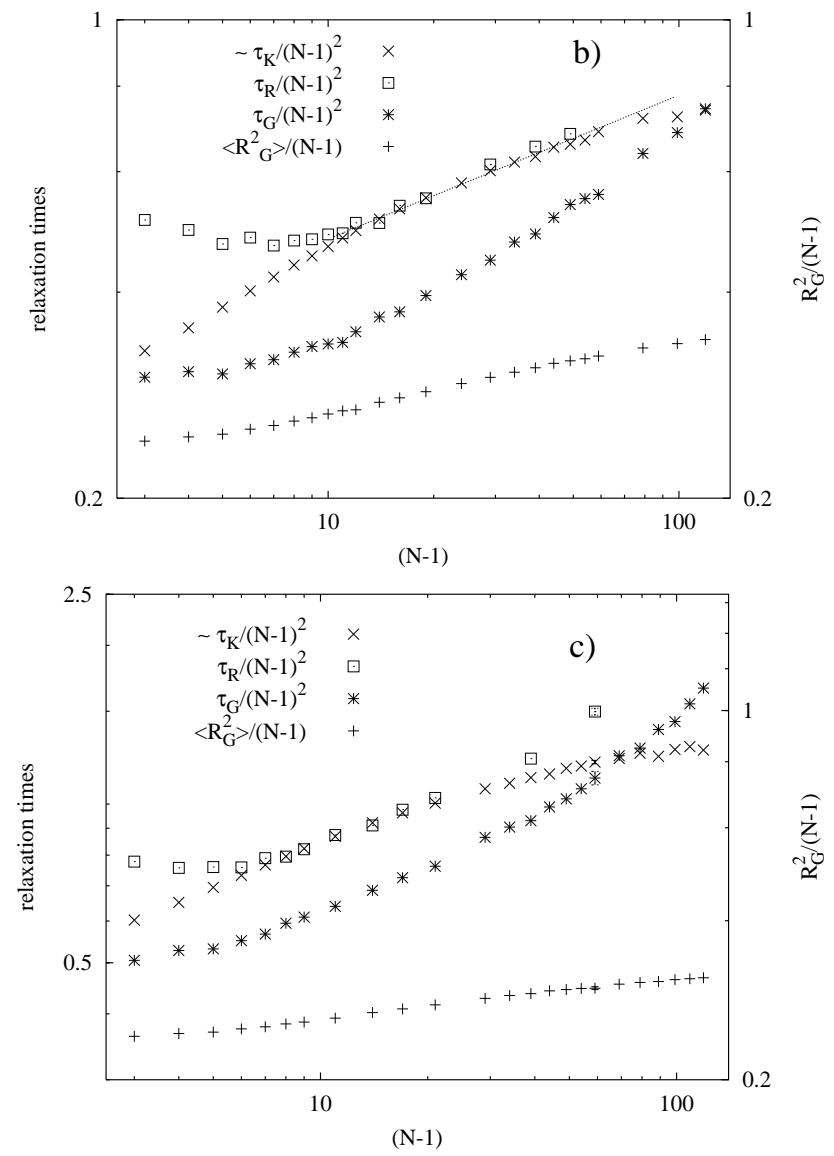

FIG. 2.

FIG. 1. 


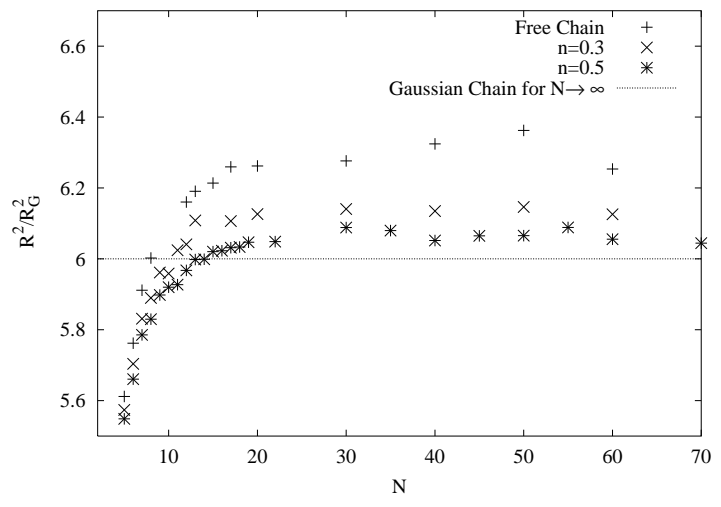

FIG. 3. 